\title{
Dental Impression Technique Using Optoelectronic Devices
}

\author{
Sinescu Cosmin ${ }^{1}$, Souman Barua ${ }^{1}$, Florin Ionel Topala ${ }^{1}$, Meda Lavinia Negrutiu ${ }^{1}$, \\ Virgil-Florin Duma ${ }^{2}$, Alin Gabriel Gabor ${ }^{1}$, Zaharia Cristian ${ }^{1}$, Adrian Bradu ${ }^{3}$, Adrian Gh. Podoleanu ${ }^{3}$ \\ ${ }^{1}$ Victor Babes University of Medicine and Pharmacy of Timisoara, Romania \\ ${ }^{2}$ Aurel Vlaicu University of Arad, Romania \\ ${ }^{3}$ University of Kent, Canterbury, UK
}

\begin{abstract}
Introduction. The use of Optical Coherence Tomography (OCT) as a non-invasive and high precision quantitative information providing tool has been well established by researches within the last decade. The marginal discrepancy values can be scrutinized in optical biopsy made in three dimensional (3D) micro millimetre scale and reveal detailed qualitative and quantitative information of soft and hard tissues. OCT-based high resolution 3D images can provide a significant impact on finding recurrent caries, restorative failure, analysing the precision of crown preparation, and prosthetic elements marginal adaptation error with the gingiva and dental hard tissues. During the CAD/CAM process of prosthodontic restorations, the circumvent of any error is important for the practitioner and the technician to reduce waste of time and material. Additionally, OCT images help to achieve a new or semi-skilled practitioner to analyse their crown preparation works and help to develop their skills faster than in a conventional way. The aim of this study is to highlight the advantages of OCT in high precision prosthodontic restorations.

Materials and methods. 25 preparations of frontal and lateral teeth were performed for 7 different patients. The impressions of the prosthetic fields were obtained both using a conventional optoelectronic system (Apolo Di, Syrona) and a Spectral Domain using OCT (Dental prototype, working at $860 \mathrm{~nm}$ ). For the conventional impression technique the preparation margins were been prelevated by gingival impregnated cords. No specific treatments were performed by the OCT impression technique.

Results. The scanning performed by conventional optoelectronic system proved to be quick and accurate in terms of impression technology. The results were represented by 3D virtual models obtained after the scanning procedure was completed. In order to obtain a good optical impression a gingival retraction cord was inserted between the prepared tooth and the gingival tissue for a better elevation of the tooth cervical margin preparation. Spectral OCT was enforced in order to observe the quality but also the advantages coming from this technology. No special preparation was performed for this operation.

Conclusion. Considering these aspects, OCT could be used as a valuable tool for dental impression technology, being non-invasive but also non-destructive on the marginal gingival tissue, in comparison with conventional optoelectronic technology where the gingival retraction cord is still mandatory.
\end{abstract}




\section{INTRODUCTION}

Optical Coherence Tomography (OCT) has multiple applications in dentistry. Such a non-invasive method could be the next emergent evaluation method to be widely adopted in the near future in dental day today activities.

\subsection{Short overview of the OCT application in dentistry}

OCT is renowned as an efficient non-invasive laser technique which can produce high spatial resolution images of biological microstructures. Similar to ultrasound, distances are measured; there are however two main differences, as in OCT sound is replaced by light and interference is used.

OCT can impact the dentistry practice; several clinical applications have been thus developed. These include a three dimensional (3D) presentation format that delivers high quality resolution with fine contrast. OCT images can be used for high precision detection of caries, tooth fractures, in periodontal disease diagnosis and oral lesion evaluation. Specific marginal enhanced visualization attributes can help detect any recurrent decay after placement of composite or gum or fixed prosthetic restorations in the mouth. Currently, OCT may be considered as an oral cancer detection aid. The extent of the lesion and tumour stage (premalignancy and malignancy status) can in principle be evaluated by OCT $[1,2]$.

In prosthodontics, the use of OCT is mostly for precision, impression technique improvisation and process step reduction. OCT is non-invasive in comparison to radiographic assessments. Therefore, OCT can be used numerous times in pre- and post-treatment phases. Additionally, OCT developments benefit prosthodontics in several respects:

- Impression taking for indirect restorations using a CAD/CAM system;

- Patient education using 3D images of OCT to explain the detailed prosthetic treatment plans;

- High-precision in marginal fit visualisation;

- Detecting micro-leakages or defects of prostheses. e.g. metal-ceramic fixed partial prostheses, metal-ceramic crowns, metal-polymer fixed partial prostheses, metal-polymer crowns, polymer and all-ceramic fixed partial prostheses, and complete dentures. Initial utilisation of en-face Time Domain (TD)-OCT technology [2] has proven the potential to replace the conventional examination of dental prostheses due to the advantages of the non-invasive and high precision attributes;

- Measuring the remaining dentin thickness (RDT) is important in the crown preparation for proper adaptability; By using OCT, the investigation of the maximum depth imaging and optical properties of the dentin near the pulp and the RDT can be explored. The dentin-pulp complex with a $1 \mathrm{~mm}$ thickness (or less) can be measured by OCT and more useful, RDT can be measured during the tooth preparation.

- Ability to observe the post-treatment infection or decay even for prosthetic works done using CAD/CAM system.

- Can help assess safe limit of tooth preparation and develop the practitioners' skills.

Although the dental practitioners use loupes or magnification in the process of crown or tooth preparation, the accuracy in tooth surface removal is practically reduced. The RDT assessment can prevent pulpal damage. Maximizing the RDT during tooth preparation is important in preventing pulpal damage. However, the related studies is still unclear the precise relationship between the pulp injury and the RDT. It has been established long time ago that deep cavities with small RDTs leave the pulp tissue at risk of preparation trauma and dental materials' chemical activity. Several studies showed that an RDT of $1 \mathrm{~mm}$ is preferable to protect the pulp tissue from the zinc phosphate and resin-modified glass ionomer materials' cytotoxic effects during the luting process. In this case, the excessive tooth preparations can damage the pulp tissue and eventually failure of the prosthodontic treatment work.

\subsection{Dental Impression Applications using OCT}

Dental impression in prosthetics represents and important process. Using classic analogical procedures based on dental impression materials leads to errors due to the different contraction coefficients. Optical impression technique can better serve this purpose, while being fast and accurate. One of the problems that could affect the optical impression technique is related to the inadequate prelevation of the margins for the prosthetic teeth preparations. The aim of this paper is to propose an optical impression guided by OCT.

\section{MATERIALS AND METHODS}

A schematic diagram of a SD-OCT system used for the current study is shown in Fig. 1. Light from a super- 
luminescent diode (SLD, central wavelength $840 \mathrm{~nm}$, spectral bandwidth $45 \mathrm{~nm}$ ), is divided by a directional coupler, $\mathrm{DC}$, into an object and a reference beam collimated by the microscope objectives $\mathrm{MO}_{1}$ and $\mathrm{MO}_{2}$ respectively, $10 \%$ of the power being directed towards the sample (approximately $800 \mu \mathrm{W}$ ) and $90 \%$ towards the reference arm. In the reference arm, light propagates through a dispersion compensation block (DCB) towards a flat mirror M that re-direct the light towards a beam splitter DC In the object arm, light is scanned laterally over the sample under test using a galvanometer scanner, $\mathrm{GX}$ and a achromatic lens $\mathrm{L}_{1}$. Light returned from the sample interferes with that originating from the reference arm in DC. Light is further detected using an in-hose spectrometer. The spectrometer consists in a transmission diffraction grating, TG, (Wasatch Photonics, $1200 \mathrm{l} / \mathrm{mm}$ ), an achromatic lens $\mathrm{L}_{2}$ of focal length $20 \mathrm{~cm}$, and a 12 bits, 2048 pixels (each 14 x $14 \square \mathrm{m}^{2}$ in size) linear CCD camera (Aviiva M2 CL). The system usually operated at an exposure time of $50 \square \mathrm{s}$ (acquisition speed 18,000 A-scans/s), which determined an acquisition rate of $18 \mathrm{~Hz}$ of Bscan OCT images when 1000 lines are used per frame. The optical path difference (OPD) between the length of the two arms of the interferometer is adjusted by altering the distance between the microscope objective MO2 and the flat mirror M.

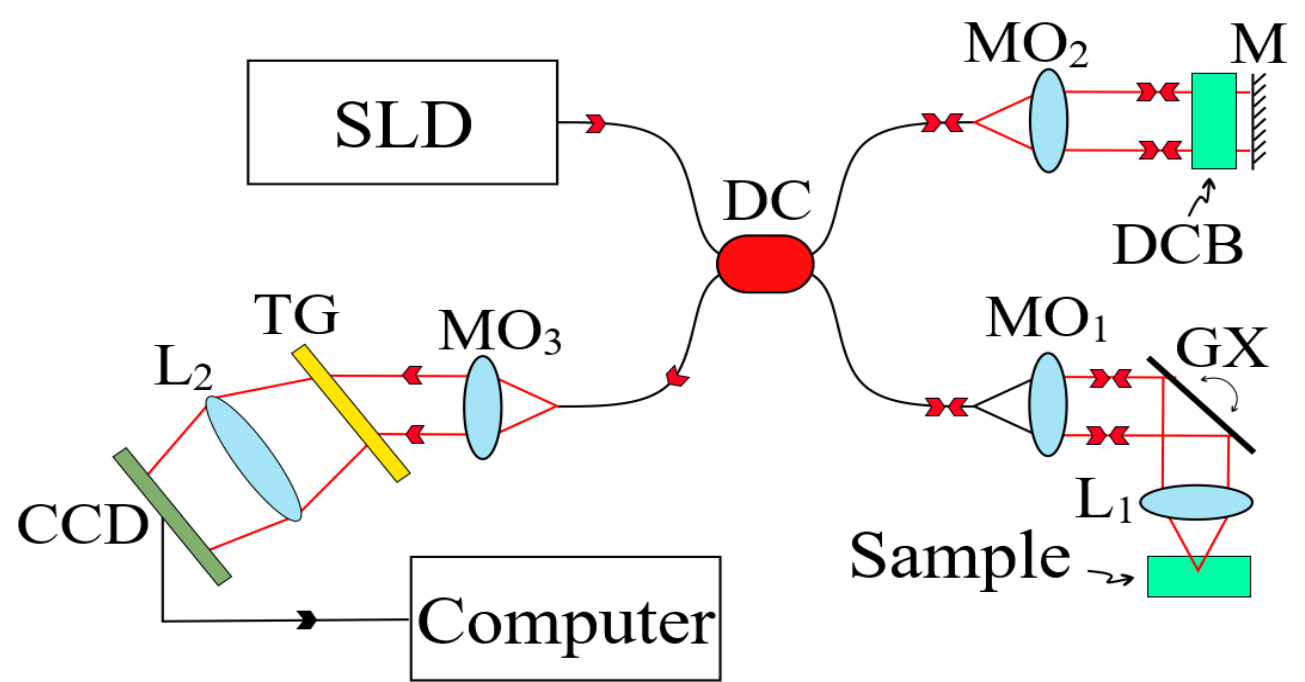

Figure 1. Schematic diagram of the OCT instrument employed for the present study. MO1,2: microscope objectives; L1,2: achromatic lenses; DC: directional coupler; SLD: luminescent diode; TG: transmission grating; GX: galvo-scanner; M: flat mirror; DCB: dispersion compensation block.

25 preparations of frontal and lateral teeth were performed in 7 different patients. The impressions of the prosthetic fields were obtained both by a conventional optoelectronic system (Apolo Di, Syrona) and an own assembled spectral OCT system working at $840 \mathrm{~nm}$. For the conventional impression technique, the preparation margins were prelevated by gingival impregnated cords. No specific treatments were performed using the OCT impression technique.

\section{RESULTS}

The scanning performed by conventional optoelectronic system proved to be fast and accurate in terms of impression technology. The results were represented by $3 \mathrm{D}$ virtual models obtained after the scanning procedure was completed (Fig. 2). In order to obtain a good optical impression, a gingival retraction cord was inserted between the prepared tooth and the gingival tissue for better elevation of the tooth cervical margin preparation.

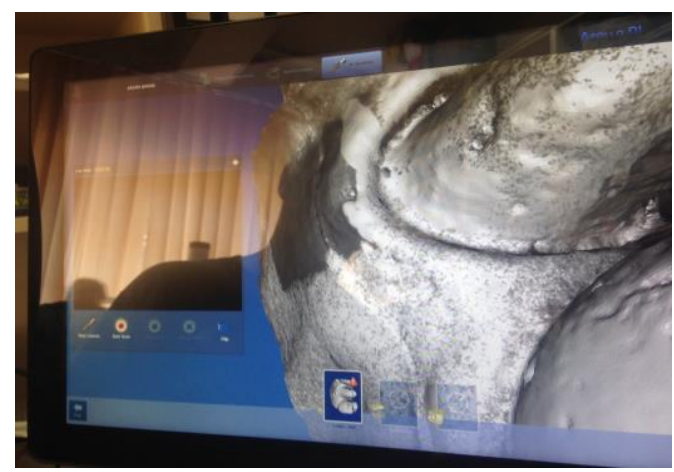

Figure 2. 3D virtual model obtained by the conventional optoelectronic system Apolo Di Sirona.

Also, the SD-OCT system was enforced for taking impression in order to observe the quality but also the 
advantages due to applying such technology (Fig. 3). No special preparation was performed for this operation.

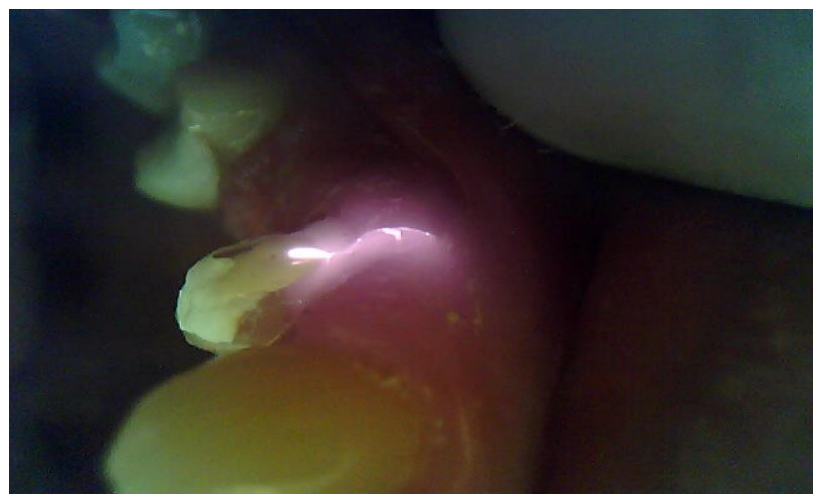

Fig. 3. The aspects from the direct intra-oral impression technology made by SD-OCT and a pilot laser (white line in the figure for a better orientation on the tissue).

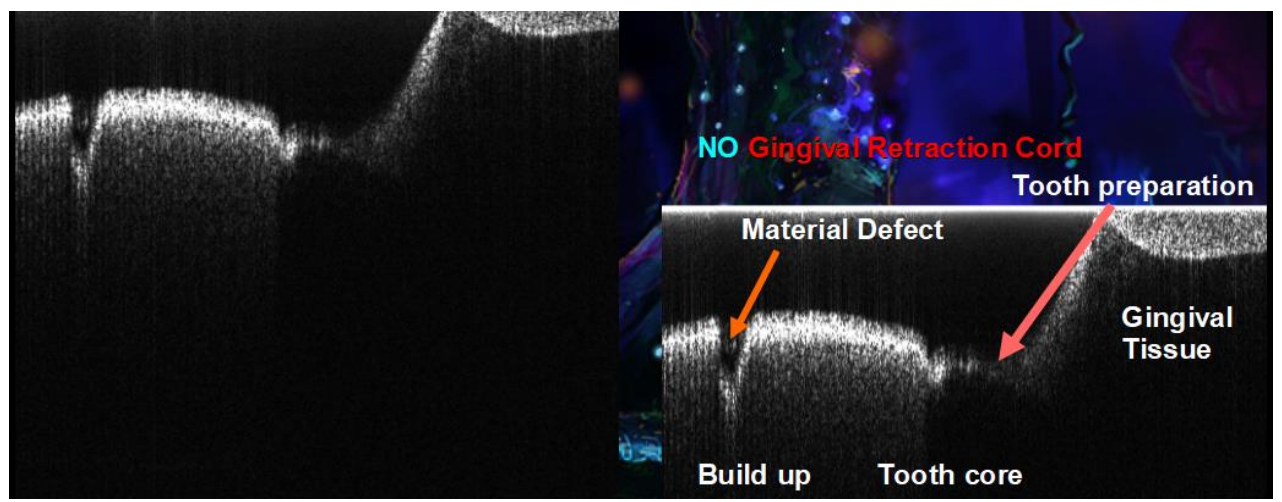

Fig. 4. The aspects of a section from the SD-OCT image that will serve for the dental impression reconstruction obtained with no gingival retraction cord.

\section{CONCLUSION}

Considering the results detailed above, OCT proves a valuable tool in the practice of dental impression technology, being non-invasive. Especially, the OCT procedure conserves the marginal gingival tissue, in comparison with the conventional optoelectronic technology where the gingival retraction cord is still mandatory.

\section{Acknowledgments}

This work was supported by the Romanian National Authority for Scientific Research, CNDI-UEFISCDI project PNIII-P2-2.1-PED-2016-1937 (http://3om-group-optomechatronics.ro/).

The authors also thank the EPSRC Grants (REBOT) EP/N019318/1 and EP/N019229/1. AP acknowledges the UBAPHODESA Marie Curie, European Industrial Doctorate 607627, the ERC Proof-of-Concept'AdaSmartRes' 754695. AP is also supported by the NIHR Biomedical Research Centre (BRC) at Moorfields Eye Hospital NHS Foundation Trust, UCL Institute of Ophthalmology and the Royal Society Wolfson Research Merit Award.

\section{References:}

1. Evans, J.W. and Tadakamalla,P. Clinical applications for Optical Coherence Tomography-Decisions in Dentistry. Decisions in Dentistry,2017.

2. Canjau,S., Todea, C., Negrutiu, M.L., Sinescu, C., Topala, I.F., Marcauteanu, C. Manescu, A. et al. Optical Coherence Tomography for Non-Invasive ex vivo Investigations in Dental Medicine - a Joint Group Experience (Review). STM J 2015 — vol. 7, No.1. DOI 10.17691/stm2015.7.1.13

3. Todea, C., Negrutiu, M.L., Balabuc, C., Sinescu, C., Topala, I.F., Marcauteanu, C. et al. Optical Coherence tomography applications in Dentistry. TMJ 2010, Vol. 60, No. 1

4. Cosmin Sinescu, Meda Lavinia Negrutiu, Adrian Bradu, Virgil-Florin Duma, and Adrian Gh. Podoleanu, "Noninvasive Quantitative Evaluation of the Dentin Layer during Dental Procedures Using Optical Coherence Tomography," Computational and Mathematical Methods in Medicine, vol. 2015, Article ID 709076, 7 pages, 2015. doi:10.1155/2015/709076 
5. Fujita, R., Komada, W., Nozaki, K. and Miura H. Measurement of the remaining dentin thickness using optical coherence tomography for crown preparation. Dental Materials Journal 2014. 33(3): 355-362.

6. Murray PE, Smith AJ, Windsor LJ, Mjor IA. Remaining dentine thickness and human pulp responses. Int Endod J 2003; 36: 33-43.

7. Pameijer CH, Stanley HR, Ecker G. Biocompatibility of a glass ionomer luting agent. 2. Crown cementation. Am J Dent 1991; 4:134-141. 